\title{
Commentary: The shunt and the precarious physiology of the shunted circulation
}

\author{
Daniel Stromberg, MD, ${ }^{\mathrm{a}, \mathrm{b}}$ and Carlos M. Mery, MD, MPH ${ }^{\mathrm{b}, \mathrm{c}}$
}
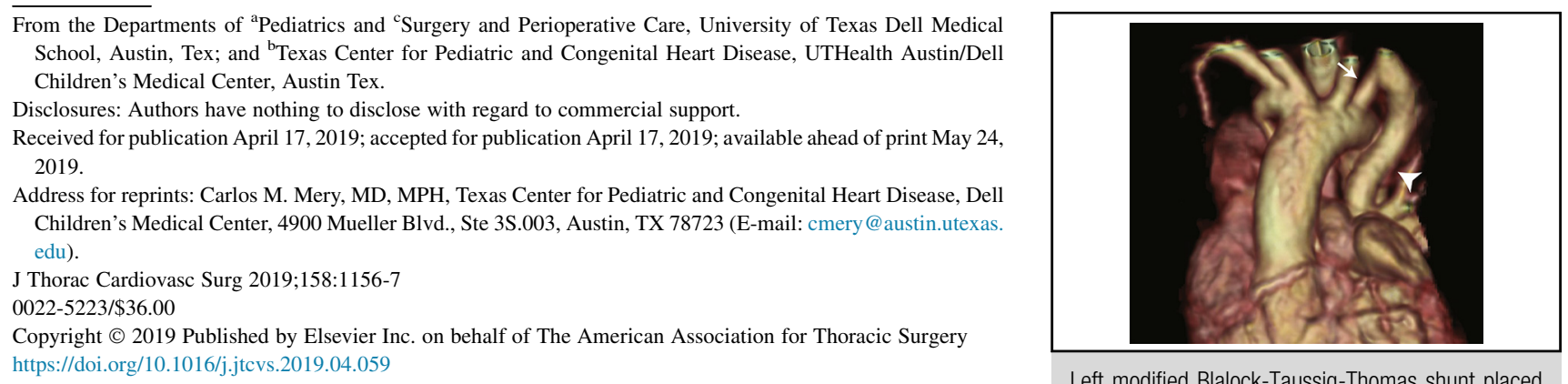

Left modified Blalock-Taussig-Thomas shunt placed through a left thoracotomy. (c) 2019 University of Texas Dell Medical School (reprinted with permission).

Complications continue to cost the lives of many children with systemic-to-pulmonary-artery (SP) shunts. SP shunts are associated with an early mortality of $10 \%$ to $15 \%$ and a rate of shunt thrombosis of $5 \%$ to $17 \%{ }^{1-5}$ Despite multiple attempts, ${ }^{2-5}$ identification of the most important factors associated with shunt complications continues to be elusive. It is likely that myriad variables interact in a highly complex and dynamic way to determine the physiologic (or nonphysiologic) behavior of the shunted parallel circulation.

Santro and colleagues ${ }^{6}$ report a series of 246 patients with a single ventricle with SP shunts. Overall mortality before second stage was $31 \%$. Acute events, defined as near-death events or cardiac arrest, occurred in $27 \%$ of patients and $53 \%$ of these patients subsequently died. These are sobering statistics that reflect the fragility and unpredictability of the shunted population.

A few important conclusions can be gleaned from the data. First, not surprisingly, once a shunted patient has an acute event, the risk of mortality increases significantly. Thus, strategies aimed at preventing an acute event by allowing early identification of impending circulatory collapse may represent the best way to improve survival. Promising approaches include creation of clinical scores based on monitoring data to alert the clinician of increasing risk of clinical decompensation, ${ }^{7}$ and the use of machine learning to create algorithms able to predict decompensation based on subtle findings before they become obvious. ${ }^{8}$

Second, immediate shunt replacement seems to be associated with better survival. The authors described milking the shunt upon re-exploration. An obvious concern is the potential embolization of thrombus to the pulmonary circulation, in particular in patients who rely on an optimal distal pulmonary vasculature and the lowest possible pulmonary vascular resistance. It is plausible that early shunt replacement may obviate some of these concerns and translate into better clinical outcomes.

Third, smaller shunts seem to be associated with a higher risk of shunt thrombosis, a finding consistent with previous studies. ${ }^{2,5}$ It is clear that one must carefully balance the risk of shunt thrombosis from a smaller shunt with the risk of overcirculation from a larger one. In trying to prevent overcirculation, the authors favored the use of smaller shunts and the wrapping of shunts whenever overcirculation was likely ( $17 \%$ of the shunts were wrapped at initial operation). We have favored the creation of SP shunts via thoracotomy (usually right) if no intracardiac intervention is necessary, because it allows placement of the shunt more distally into the subclavian artery (Figure 1). The subclavian artery then becomes the regulator of shunt flow, rendering shunt diameter almost irrelevant. This technique permits use of a larger shunt (potentially reducing the risk of thrombosis) while limiting overcirculation. McKenzie and colleagues ${ }^{2}$ confirmed that creation of the shunt via thoracotomy and into the subclavian artery (rather than the innominate artery) was independently associated with improved survival.

The study by Santro and colleagues ${ }^{6}$ enhances our understanding of potentially modifiable factors that improve 


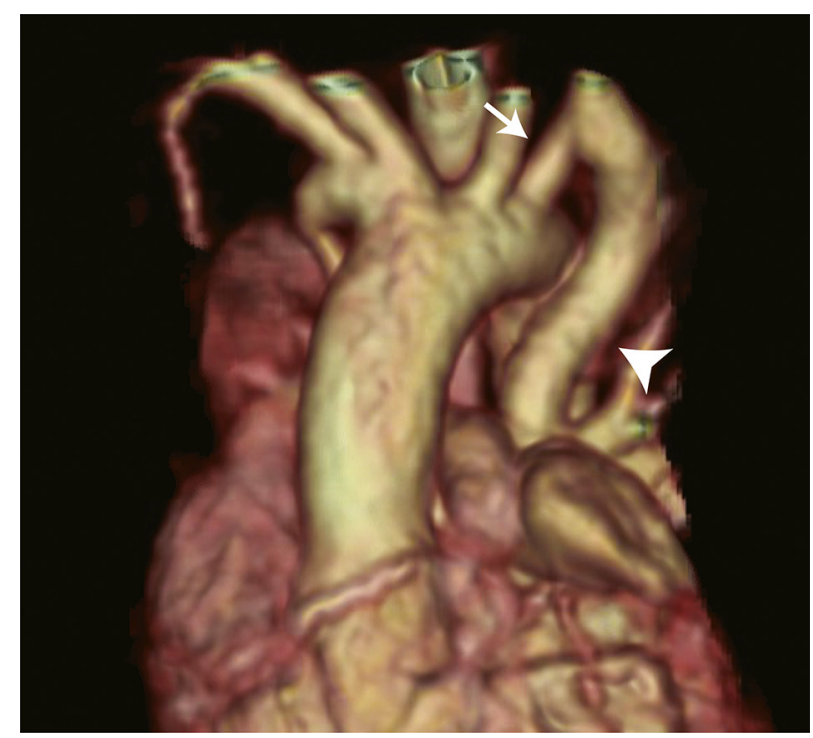

FIGURE 1. Computed tomography of a patient with heterotaxy who underwent placement of a left-modified Blalock-Taussig-Thomas shunt via a left thoracotomy. A thoracotomy approach allows placement of a large shunt (arrowhead) completely within the subclavian artery (arrow), which acts as the regulator of shunt flow. (C) 2019 University of Texas Dell Medical School (reprinted with permission).

survival. Nonetheless, given the complex multifactorial nature of shunt failure, a multiprong strategy that involves a combination of optimal surgical technique, informed postoperative anticoagulation, careful control of systemic and pulmonary vascular resistances, and early detection of clinical decompensation will be paramount to reducing mortality in this fragile population.

\section{References}

1. Wessel D, Berger F, Li JS, Dähnert I, Rakhit A, Fontecave S, et al. Clopidogrel in infants with systemic-to-pulmonary-artery shunts. N Engl J Med. 2013;368: 2377-84.

2. McKenzie ED, Khan MS, Samayoa AX, Vener DS, Ishak YM, Santos AB, et al The Blalock-Taussig shunt revisited: a contemporary experience. J Am Coll Surg. 2013;216:699-706.

3. Saini A, Joshi AD, Cowan KM, Gatewood CW, Kumar STK, Allen J, et al. High acetylsalicylic acid dosing in infants after modified Blalock-Taussig shunt. Cardiol Young. 2019;29:389-97.

4. Patregnani JT, Sochet AA, Zurakowski D, Klugman D, Diab Y, Berger JT, et al Cardiopulmonary bypass reduces early thrombosis of systemic-to-pulmonary artery shunts. World J Pediatr Congenit Heart Surg. 2018;9:276-82.

5. Vitanova K, Leopold C, von Ohain JP, Wolf C, Beran E, Lange R, et al. Reasons for failure of systemic-to-pulmonary artery shunts in neonates. Thorac Cardiovasc Surg. 2019;67:2-7.

6. Santro T, d'Udekem Y, Zannino D, Hobbes B, Konstantinov IE, Brizard C, et al Determinants of acute events leading to mortality after shunt procedure in univentricular palliation. J Thorac Cardiovasc Surg. 2019;158:1144-53.e6.

7. Ruiz VM, Saenz L, Lopez-Magallon A, Shields A, Ogoe H, Srinivasan S, et al Early prediction of critical events for infants with single-ventricle physiology in critical care using routinely collected data. J Thorac Cardiovasc Surg. February $21,2019$.

8. Rusin CG, Acosta SI, Shekerdemian LS, Vu EL, Bavare AC, Myers RB, et al. Prediction of imminent, severe deterioration of children with parallel circulations using real-time processing of physiologic data. J Thorac Cardiovasc Surg. 2016;152: $171-7$. 